TOC Graphic

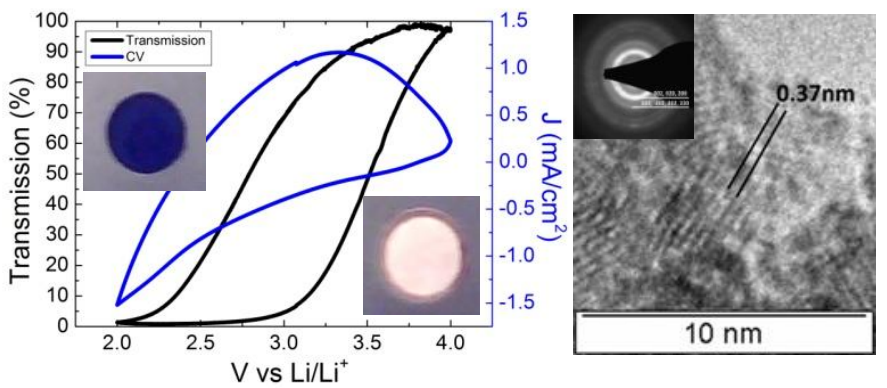




\title{
Scalable synthesis of improved nanocrystalline, mesoporous tungsten oxide films with exceptional electrochromic performance
}

\author{
Chi-Ping Li,${ }^{\mathrm{a}, \mathrm{b}}$ Chaiwat Engtrakul, ${ }^{\mathrm{b}}$ Robert C. Tenent, ${ }^{\mathrm{b}}$ and Colin A. Wolden ${ }^{\mathrm{a}, *}$ \\ ${ }^{a}$ Department of Chemical and Biological Engineering and the Materials Science Program, \\ Colorado School of Mines, Golden, CO 80401, USA \\ ${ }^{\mathrm{b}}$ National Renewable Energy Laboratory, Golden, CO 80401, USA
}

\begin{abstract}
Templated sol gel chemistry provides a versatile approach to introduce order and porosity into nanostructured materials. However conventional evaporation induced self assembly techniques are not easily scaled to produce films with sufficient thickness over large areas at the throughput required by electrochromic windows. Here we demonstrate that the principles of sol gel chemistry may be deployed using ultrasonic spray deposition (USD) for scalable synthesis of nanocrystalline $\mathrm{WO}_{3}$ films with unrivaled electrochromic performance. Systematic manipulation of sol chemistry enabled the production of mesoporous films with high specific surface area $\left(>100 \mathrm{~m}^{2} / \mathrm{g}\right)$, mean pore sizes of $\sim 5 \mathrm{~nm}$, and narrow pore size distributions. Film thickness is found to be proportional to the sol concentration and number of spray passes, and various combinations are shown to produce films capable of modulating $>98 \%$ of incident solar radiation in the visible spectrum $(450-900 \mathrm{~nm})$. Elimination of haze enables full transmission in the bleached state, while the broadband coloration is attributed to the exceptionally high charge density $\left(>120 \mathrm{mC} / \mathrm{cm}^{2}\right)$. The materials have good switching speeds which improve with specific surface area, and the long term durability is promising.
\end{abstract}

Keywords: electrochromic, mesoporous, sol gel, templated, tungsten oxide, ultrasonic spray deposition

* Corresponding author. E-mail address: $\underline{\text { cwolden@mines.edu }}$ 


\section{Introduction}

Buildings account for $40 \%$ of energy expenditures in the US, more than any other sector including transportation and industry [1]. Windows are responsible for a significant fraction of energy loss in buildings, strongly influencing cooling, heating and lighting requirements. Smart windows employing electrochromics (EC) have the potential to significantly reduce the US energy footprint by up to 5 quads ( 1 quad $=10^{15}$ BTU) by controlling solar heat gain and lighting through modulation of their optical characteristics (i.e. transmittance, reflectance). The optical transparency of electrochromic materials such as tungsten oxide is reversibly altered due to the insertion/extraction of light ions such as $\mathrm{Li}^{+}$or $\mathrm{H}^{+}$in response to a small applied voltage [2]. There has been significant progress in the forty years since Deb's pioneering studies $[3,4]$, but current EC applications remain limited to niche markets such as dimmable rear view mirrors and the windows of Boeing's Dreamliner. Further improvements in both performance and cost reduction are required to enable widespread deployment of this technology.

In his recent critical review, Granqvist identified a number of hurdles that must be overcome in order to enable large scale manufacturing of EC smart windows [5]. Prominent among them was that EC films must have nanoporosity over their entire area, which may require that films are produced under non-standard conditions using techniques that are amenable to large-scale manufacturability. Tungsten oxide films with a mesoporous, nanocrystalline morphology are ideal for electrochromic applications [6]. The crystalline phase is preferred over amorphous material for stability concerns $[6,7]$, while high specific area is required for efficient ion intercalation. Templated sol-gel chemistry approaches have been used to produce mesoporous $\mathrm{WO}_{3}$ with impressive 
electrochromic properties [8-15]. For example, the highest optical modulation reported to date of $\Delta \mathrm{T}=85 \%$ was achieved for $\mathrm{WO}_{3}$ films deposited using sol gel chemistry $[14,15]$, a value that is superior to leading reports from sputter deposited films [16]. Solutionbased chemical synthesis also offers the promise of reduced manufacturing costs relative to current state-of-the-art vacuum deposited electrochromics [17]. However, the techniques employed to produce these results such as dip coating $[8,11,14]$, spin coating [9], or film casting $[10,15]$ are generally quite slow and not easily amenable to large scale, in line manufacturing. As such, commercial production of smart windows continues to rely on sputtered $\mathrm{WO}_{3}$, largely due to its demonstrated reliability for coating large area substrates $[2,4]$.

Our group has been exploring the potential of ultrasonic spray deposition (USD) for the scalable fabrication of EC devices. Advantages of USD include low capital requirements and high materials utilization. Moreover, the use of benign solvents such as water and alcohol allows processing to be conducted under ambient conditions. Spray pyrolysis has been demonstrated for the production of high quality transparent electrodes [18] as well a nickel oxide [19], a leading counter electrode in the electrochromic stack [2]. As such one could envision employing USD for in-line production of smart windows. One approach for the formation of mesoporous, nanocrystalline films is to first produce crystalline nanoparticles, and then assemble them into a mesoporous network [20, 21]. This strategy inspired our initial USD approach which employed a two step process. First, crystalline $\mathrm{WO}_{3}$ nanoparticles (NP) were produced by hot-wire chemical deposition (HWCVD) [22]. The resulting NPs were then suspended in an ethanol solution and sprayed to form mesoporous films using USD [23]. As expected, the electrochromic 
performance improved as the NP size was reduced [24], but it proved difficult to reduce the NP size beyond $15 \mathrm{~nm}$ using HWCVD or envision scaling NP production to the quantities required for large scale manufacturing. More recently we demonstrated that templated sol-gel chemistry could be adapted to USD [25]. In this work a conventional sol-gel chemistry was used [26], with the exception that the sol was applied by USD onto an unheated substrate instead of by spin coating. This may be considered a variant of evaporation induced self assembly (EISA) in which evaporation occurs from individual droplets as opposed to from a film, accelerating the process. It was found that the postdeposition calcination step was critical, and films with fast switching time $(<10 \mathrm{~s})$ were obtained by rapid annealing technique in which samples were place directly on a hot plate maintained at $350{ }^{\circ} \mathrm{C}$ after the gelation step [25].

Fig. 1 displays the transmission spectra of a mesoporous $\mathrm{WO}_{3}$ film produced by USD previously in both its bleached and colored state as well as its optical modulation [27]. This level of performance is representative of current state-of-the-art material [5, 28]. The optical modulation reaches $75 \%$ in the near infrared but decreased as the wavelength is reduced, dropping precipitously below $600 \mathrm{~nm}$. Transmission losses in the bleached state are attributed to haze, which arises due to scattering from either surface roughness or the presence of voids or agglomerates within the film [29]. The contribution of these scattering losses increase as the wavelength is reduced. In the colored state the transmission is controlled by the degree of ion intercalation. We found that the electrochromic performance correlates with the nanostructure and the specific surface area of the nanocrystalline films [27]. However the specific surface area of the best material in our previous study was just $18 \mathrm{~m}^{2} / \mathrm{g}$, whereas values as high as $145 \mathrm{~m}^{2} / \mathrm{g}$ have 
been reported for mesoporous $\mathrm{WO}_{3}$ synthesized from conventional sol-gel processes [12], so substantial room for further improvement remains.

It is well known that the shape and size of the mesoporous networks produced by soft templating may be directed through the control of parameters such as sol composition. The volume fraction of the block copolymer can be used for the rationale control of nanostructure [30], and optimization of this critical parameter has been used successfully to control porosity and increase specific surface area in a number of different material systems $[10,31,32]$. For well-studied materials such as silica and titania the relationship between sol chemistry and resulting nanostructure has been extensively explored, and comprehensive phase diagrams are available [33,34]. Such systematic studies have not been reported for $\mathrm{WO}_{3}$. Fig. 2 provides a ternary phase diagram for this sol composition, which is comprised of $\mathrm{WCl}_{6}$ and the commonly used triblock copolymer Pluronic P123 dissolved in ethanol. Our previous USD work employed a sol that was $~ 95 \mathrm{wt} \%$ ethanol, due to concerns about maintaining low viscosity to ensure a well-dispersed mist using the ultrasonic nebulizer. Fig. 2 includes sol compositions previously reported in the literature for this chemistry $[8,35-37]$. Though none of these studies reported on the sensitivity to sol composition, presumably there was some level of optimization and these points provided guidance for the present study. Although there is substantial variation, the previous reports all used sols with both higher concentration and elevated $\mathrm{WCl}_{6}: \mathrm{P} 123$ ratios. Comparison of these studies also suggests that the pore size decreased as the sol concentration was increased. Within this region we found that optimum performance was obtained at the compositions indicated by the squares in Fig. 2. The $\mathrm{WCl}_{6}: \mathrm{P} 123$ ratio in each case was 0.595 by mass, an increase from the value of 0.25 used in our previous 
work [25]. With the $\mathrm{WCl}_{6}: \mathrm{P} 123$ ratio fixed, the impact of the total concentration in ethanol was also examined. In this work we examined sols with $\mathrm{WCl}_{6}$ concentrations of $1.5,2.25$ and $3 \mathrm{mM}$, which are denoted on the diagram and throughout this work as $1 \mathrm{C}$, $1.5 \mathrm{C}$ and $2 \mathrm{C}$, respectively. The viscosity of the sol increased linearly with concentration, and it was found that the $2 \mathrm{C}$ concentration level was the practical limit for producing uniform films without clogging of the ultrasonic nebulizer or degradation of mist quality. However, we note that during USD process the sol is further concentrated along the pathway indicated by the arrow in Fig. 2 due to ethanol evaporation.

The sol concentration significantly impacts the morphology and nanostructure of the tungsten oxide films, as well as their electrochromic performance. We first describe the physical characteristics of the resulting films as a function of sol concentration, confirming their mesoporous, nanocrystalline nature through electron microscopy, selected area diffraction, and physisorption studies. Next we demonstrate that the thickness of the resulting films is directly proportional to the product of the sol concentration and the number of passes under the nebulizer. This feature was used to optimize the electrochromic performance as measured by cyclic voltammetry and step potential experiments. Herein we describe the synthesis of the first tungsten oxide films capable of displaying $100 \%$ optical modulation, and show that this response is achieved across most of the visible spectrum. The importance of nanostructure is evident in measurements of switching time. We conclude by demonstrating the intrinsic durability of these materials under extended cycling. 


\section{Materials and Methods}

\subsection{Preparation of mesoporous nanocrystalline tungsten oxide films}

Triblock polymer Pluronic P123 (poly (ethylene oxide)-b-poly (propylene oxide)-bpoly (ethylene), $\mathrm{EO}_{20} \mathrm{PO}_{70} \mathrm{EO}_{20}$ ), anhydrous ethanol and tungsten hexachloride $\left(\mathrm{WCl}_{6}\right.$ ) were all purchased from Sigma-Aldrich and used without further purification. An inert glovebox was used for reagent storage and sol preparation, due to the moisture sensitivity of these components. Sol solution preparation began by separately dissolving 1, 1.5 and 2 $\mathrm{g}$ of the triblock copolymer P123 in $10 \mathrm{ml}$ anhydrous ethanol ( $\geq 99.5 \%)$ for $1 \mathrm{C}, 1.5 \mathrm{C}$ and 2C concentration levels, respectively. The sol was completed by adding $0.595,0.8925$ and $1.19 \mathrm{~g}$ of $\mathrm{WCl}_{6}(\geq 99.9 \%)$ to the $1 \mathrm{C}, 1.5 \mathrm{C}$ and $2 \mathrm{C}$ triblock copolymer/ethanol solution and followed by 12 hours of stirring to produced sols that were then used for ultrasonic spray deposition.

The stable sols described above were transferred to a syringe and delivered to the ultrasonic spray nozzle at a flow rate of $0.25 \mathrm{ml} / \mathrm{min}$ using Fluid Metering Inc. VMP TRI Pulseless "Smoothflow" pump. The ultrasonic spray system was obtained from Sono-Tek Corporation and consisted of a model $8700-120$ spray head that operated at a frequency of $120 \mathrm{kHz}$. The spray nozzle had a 0.230 in. diameter conical tip and 0.015 in. diameter orifice that was fitted with the impact system for gas-driven spray delivery. The atomized mist was entrained in a stream of nitrogen whose flowrate was fixed at 6.9 standard liters/minute using an electronic mass flow controller (Omega FMA 1818). This aerosol was directed onto FTO-coated glass (TEC-15, Pilkington) positioned $5 \mathrm{~cm}$ below the nozzle under ambient conditions. Before deposition, all substrates were cleaned with an isopropanol-soaked clean-room wipe, blown dry with nitrogen, and then placed in an 
oxygen plasma (800 mtorr, $155 \mathrm{~W}$ ) for 5 minutes. Samples were mounted on a computer controlled stage which rastered them through the deposition zone for the desired number of spray passes, producing uniform, iridescent blue films. Samples were then transferred into a chamber saturated with water vapor for hydrolysis.

The final step is calcination, where samples are heated in air to remove residual solvent and the polymer template while completing the oxidation and crystallization of the $\mathrm{WO}_{3}$. Samples were placed directly onto a hot plate set at maintained at $350{ }^{\circ} \mathrm{C}$. After 1 hour on the hot plate the samples were removed and allowed to naturally cool down to room temperature.

\subsection{Characterization}

Samples for transmission electron microscopy (TEM) and selected area diffraction (SAD) were produced by suspending scraped tungsten oxide powder in ethanol using sonication and placing a drop on a TEM grid and allowing the solvent to evaporate. The nanoscale morphology was examined by using a Philips CM200 transmission electron microscope, and SAD patterns were collected using $200 \mathrm{kV}$ electrons. FESEM characterization of the films was performed on a JEOL JSM-7000F microscope operating at $2 \mathrm{keV}$. The crystallinity of final tungsten oxide films were assessed by X-ray diffraction (XRD) using $\mathrm{CuK} \alpha$ radiation over a range of $2 \theta=20$ to $65^{\circ}$ using $0.05^{\circ}$ steps. $\mathrm{N}_{2}$ physisorption was performed using a Micrometrics ASAP 2020 after samples were degassed at $250{ }^{\circ} \mathrm{C}$ under vacuum for $4 \mathrm{~h}$. 


\subsection{Electrochromic performance}

Electrochromic performance was evaluated by cycling films in an electrolyte composed of $1 \mathrm{M} \mathrm{LiClO}_{4}$ dissolved in propylene carbonate in a test cell housed in an Arpurged glovebox. Cyclic voltammetry and potential cycling measurements were made using a BioLogic VMP3 multichannel potentiostat. Cyclic voltammetry was performed using a $20 \mathrm{mV} / \mathrm{s}$ scan rate between 2 and $4 \mathrm{~V}$ vs. $\mathrm{Li} / \mathrm{Li}^{+}$. Cyclic voltammograms shown in this work are from the $2^{\text {nd }}$ or $3^{\text {rd }}$ cycle when performance had stabilized. Switching kinetics was measured using chronoamperometric cycling between 1.7 and $4.2 \mathrm{~V}$ vs. $\mathrm{Li} / \mathrm{Li}^{+}$, and the switching speed was defined as the time required to achieve a $75 \%$ change in the absolute transmission. Long term durability studies were performed by cycling at a constant current density of $39 \mu \mathrm{A} / \mathrm{cm}^{2}$ between 2 to $3 \mathrm{~V} \mathrm{vs}$. Li/ $/ \mathrm{Li}^{+}$.

A diode laser (670 nm) coupled to a detector (Thor Labs, Inc. DET100A) was used to collect optical transmission in direct registry during cycling. For analysis of the full transmission spectra the laser and detector were replace by a broadband halogen light source and an Ocean Optics USB 4000 fiber optic spectrometer, respectively. In both cases the contribution of a clean FTO substrate placed in the electrolyte solution was background subtracted, so that the reported optical response reflects only contributions from the $\mathrm{WO}_{3}$ film. In addition, still images of the transmission change were acquired by illuminating the cell with a standard incandescent flashlight and recording the transmission with a fiber-optic based camera (General Tools Model DCS050). 


\section{Results and Discussion}

\subsection{Physical characterization of mesoporous nanocrystalline films}

Fig. 3a,b displays cross-section and plan view scanning electron micrographs of a 1 micron thick film that was deposited at the intermediate $1.5 \mathrm{C}$ sol. From the cross section images the mesoporous structure is clearly evident, and the plan view image shows that the surface is quite smooth. In addition it is evident that the films are very homogeneous, and the absence of mesoscale clusters or voids dramatically improves the optical quality of these films. Fig. 3c shows a photograph of this sample comparing it with an uncoated FTO/glass substrate. The films display perfect uniformity and the issue of haze, which is commonly observed in solution-processed films, is completely eliminated. This is consistent with the smooth, homogeneous morphology observed in the SEM images (Fig. 3b). As shown below, this feature enables near perfect transmission in the bleached state. Further photographic evidence displaying the scalability and uniformity enabled by this process are provided in the supplementary information Appendix A (Fig. A.1).

Increasing sol concentration was found to improve the nanostructure of these films. There is no evidence of long range order in these materials, but periodicity is not a prerequisite for high performance [38]. Critical metrics typically include crystallinity, porosity, pore size, and specific surface area. Fig. 4 displays transmission electron microscopy (TEM) images and selected area diffraction (SAD) patterns obtained from mesoporous tungsten oxide deposited at the three different concentration levels. The nanocrystalline nature of the material is confirmed by TEM imaging, as lattice fringes are readily observed at all three conditions. The lattice spacing of $0.37 \mathrm{~nm}$ and the indexing of the associated $\mathrm{SAD}$ patterns confirms that $\mathrm{WO}_{3}$ is present as the monoclinic $\gamma$-phase, 
which is the most commonly observed form of tungsten oxide [36]. As the sol concentration increases the primary particle size decreases from around 5-6 $\mathrm{nm}$ at $1 \mathrm{C}$ to 3-4 $\mathrm{nm}$ at $2 \mathrm{C}$. These qualitative observations of crystallite size are confirmed by the SAD patterns. At the baseline concentration, the crystalline domains are sufficiently large that distinct spots dominate the diffraction patterns. As the concentration increased the intensity of these spots is attenuated, and at $2 \mathrm{C}$ only rings are observed in the $\mathrm{SAD}$ patterns. Wide angle X-ray diffraction patterns further confirm the phase and the nanocrystalline nature of these materials (Fig. A.2).

The structural changes described above were accompanied by systematic improvements in the specific surface area of the mesoporous films. Fig. 5a compares a $\mathrm{N}_{2}$ physisorption isotherm obtained from material produced in this work at $1.5 \mathrm{C}$ with the best material produced in our previous study [25]. Isotherms obtained at the $1 \mathrm{C}$ and $2 \mathrm{C}$ concentration levels were similar and are provided in the supporting information (Fig. A.3). The shapes of the isotherms from the material produced in this work correspond to type IV of the BET classification, characteristic of mesoporous materials [39]. The dramatic increase in both total volume and the level of splitting between the adsorption/desorption branches illustrate the profound differences between the two materials. Fig. 5b compares the pore size distributions extracted from the physisorption curves. Our previous material displayed a very broad pore size distribution ranging from $5-50 \mathrm{~nm}$, a mean pore size of $18 \mathrm{~nm}$, and a specific surface area of $18 \mathrm{~m}^{2} / \mathrm{g}$ [25]. The mesoporous material produced in this work is characterized by much smaller pores with a tighter distribution and significantly enhanced specific surface area. At the baseline concentration (1C) the mean pore size was $7 \mathrm{~nm}$, and the resulting specific surface area 
was $75.8 \mathrm{~m}^{2} / \mathrm{g}$. At the concentration level of $1.5 \mathrm{C}$ the mean pore size remained unchanged, but the distribution narrowed and the specific surface area increased to 114 $\mathrm{m}^{2} / \mathrm{g}$. At the highest concentration (2C) the mean pore size decreased to $5 \mathrm{~nm}$ as the density of small pores was enhanced, and the specific surface area was further increased to $120 \mathrm{~m}^{2} / \mathrm{g}$. These physisorption results are perfectly consistent with the trends observed in TEM, SAD, and XRD. The specific areas achieved in this work are comparable to the highest values ever reported for mesoporous tungsten oxide.[8, 12] The variations in physical properties are reflected in the electrochemical measurements described below.

\subsection{Electrochromic Characterization}

It was found that the film thickness was proportional to the product of sol concentration $(\mathrm{C})$ employed and the number of passes $(\mathrm{P})$ made through the deposition zone. Fig. 6 plots the film thickness as measured by cross sectional SEM images (Fig. A.4) as function of the product of these two variables $(\mathrm{C} \times \mathrm{P})$. For example, the data at $\mathrm{C}$ $x \mathrm{P}=6$ was obtained from three films deposited using $1 \mathrm{C} \times 6 \mathrm{P}, 1.5 \mathrm{C} \times 4 \mathrm{P}$, and $2 \mathrm{C} \times 3 \mathrm{P}$, respectively. A strong linear relationship between film thickness and the product $\mathrm{C} x \mathrm{P}$ was observed, which facilitated manipulation of the extrinsic electrochromic response. The optical modulation of these films was recorded in registry during cyclic voltammetry experiments. Fig. 7 displays the cyclic voltammogram $(\mathrm{CV})$ and the associated optical response from a film deposited under optimal conditions, and Fig. 6 summarizes the optical modulation achieved during cyclic voltammetry evaluation of an array of films of varying thickness deposited using the three sol concentrations. For all sol concentration levels it was found that the optical modulation was maximized at $\mathrm{C} \times \mathrm{P}=6$, which 
corresponds to films approximately 1 micron thick (Figs. 6, A.4). At this condition the optical modulation reaches the theoretical limit of $100 \%$, setting a new and ultimate standard for this material. The CV presented in Fig. 7 was obtained from a film produced using $1.5 \mathrm{C}$ x $4 \mathrm{P}$, and very similar results are obtained at both the $1 \mathrm{C}$ and $2 \mathrm{C}$ levels (Fig. A.5). For films deposited at $\mathrm{C} \times \mathrm{P}<6$ the optical modulation declines because the films are too thin and the transmission cannot reach $0 \%$ in the colored state. Likewise, films deposited at $\mathrm{C} \times \mathrm{P}$ values greater than 6 the films are too thick, and cannot reach $100 \%$ transmission in the bleached state. Fig. 6 contains data obtained at all three concentration levels, and though there is some scatter in the data, the basic trends with thickness are quite consistent.

The optical response reported above was recorded by a laser operating at $670 \mathrm{~nm}$. To assess the full spectral response the laser and photodiode were replaced by a broadband halogen source and a fiber-optic based spectrometer, respectively. Fig. 8 shows the transmission spectra obtained in both the colored and bleached states from a film deposited using 1.5C x 4P. In the bleached state perfect transparency is obtained across the full spectral range, with any deviation associated with noise induced by the background subtraction procedure. This is attributed to the elimination of haze as discussed above (Fig. 3). Likewise, the films are completely opaque at wavelengths $>550$ $\mathrm{nm}$. As the wavelength is further reduced a small amount of blue light is transmitted, but remains $<8 \%$ at $450 \mathrm{~nm}$. The significant improvement in broadband optical modulation has important implications for the energy saving potential of smart windows. By integrating the product of the optical modulation with the AM1.5 solar spectrum it is 
found that these tungsten oxide films modulate $>98 \%$ of the solar radiation present over the spectrum examined.

Aesthetics are an important consideration in electrochromic applications, so the optical response was also quantified by illuminating one side of the testing cell with a standard incandescent flashlight and monitoring the transmission with a fiber optic-based camera on the other. Photographs of the illuminated test cell with tungsten oxide in its colored and bleached states are provided as insets to Fig. 8. These agree well with the spectra, as white light is observed in the transparent state and dark blue in the opaque, reflecting the low wavelength transmission shown in the spectra. The photographs also provide a testament to the uniformity, as there is no variation in appearance across the 1 $\mathrm{cm}$ diameter site glass. Again the performance for films deposited at the other concentration levels displayed nominally identical spectra, examples of which are contained in the supporting information Appendix A (Fig. A.6).

As shown above, the electrochromic performance during cyclic voltammetry was nominally identical at the three concentration levels. The intercalation rate of lithium ions during the $\mathrm{CV}$ experiments is relatively slow, and as such the coloration efficiency reflects the total charge capacity of the material and not the kinetics. Chronoamperometry experiments employing alternating step potentials with a period of $120 \mathrm{~s}$ were used to assess the switching dynamics the films. Fig. 9 displays the response of selected films at each of the three concentration levels. In contrast to the CV results, the dynamic response is sensitive to the differences in nanostructure discussed above. Note that the degree of voltage modulation $\left(1.7-4.2 \mathrm{~V}\right.$ vs. $\left.\mathrm{Li} / \mathrm{Li}^{+}\right)$used in these experiments was greater than in the CV studies $\left(2-4 \mathrm{~V}\right.$ vs. $\left.\mathrm{Li} / \mathrm{Li}^{+}\right)$, which is why $100 \%$ optical modulation is obtained in 
films that are thinner than $\mathrm{C} \times \mathrm{P}=6$ value that was found to be optimal in the $\mathrm{CV}$ studies (Fig. 6). In all cases the rate of coloration is fast, and it is the bleaching step that limits switching speed. In these step potential experiments the transmission changes during bleaching are controlled by the rate of $\mathrm{Li}^{+}$extraction from $\mathrm{WO}_{3}$ and transport out of the film. As such the impact of both nanostructure and total film thickness is evident. At the $1 \mathrm{C}$ level, the optimum number of passes was between 4 and 5 . In the colored state these films were completely opaque, but could only reach $\sim 90 \%$ transparency during the $120 \mathrm{~s}$ bleaching step. At the intermediate concentration $1.5 \mathrm{C}$ × $2 \mathrm{P}$ was observed to produce the optimal thickness, obtaining $96 \%$ optical modulation. At the highest concentration films made using 2C x 2P achieved $100 \%$ optical modulation. Films deposited at 2C x 1P were insufficiently opaque under coloration, while 2C x 3P films were too thick and limited by the response time.

Switching speed was quantified by the time required to modulate the absolute transmission by $75 \%$. For coloration, which is the thermodynamically favored process, the switching times of three different concentration levels are very fast, around just 3 seconds. However, the bleaching time is sensitive to nanostructure, decreasing with sol concentration from $67 \mathrm{~s}$ at $1 \mathrm{C}$ to $27 \mathrm{~s}$ at $2 \mathrm{C}$. Both the switching times and the maximum level of optical modulation $(90,96,100 \%)$ achieved in these step potential experiments correlates exceptionally well with the specific surface area $\left(76,114,120 \mathrm{~m}^{2} / \mathrm{g}\right)$ obtained from the three concentration levels, reflecting the importance of this parameter on the rate of $\mathrm{Li}^{+}$extraction.

Coloration efficiency (CE), which is conventionally defined as the change in optical density (OD) per unit of charge density $(\Delta \mathrm{Q})$ intercalated into the tungsten oxide, is an 
important parameter for comparing the intrinsic performance of electrochromic materials [6]. The CE was calculated from the chronoamperometry experiments using the following equations:

$$
\begin{aligned}
& C E=\frac{O D}{\Delta Q} \\
& O D=\log \left(\frac{T_{b}}{T_{c}}\right) \\
& \Delta Q=\jmath J d t
\end{aligned}
$$

where $T_{b}$ and $T_{c}$ refer to the transmittance of the layer in its bleached and colored states, respectively, and $J$ is the current density. Fig. 10 plots the optical density versus charge density for films fabricated at the three concentration levels. The CE is extracted from the slope of the fit to the linear region of the curves. The CE values for all samples analyzed were on the order of $40 \mathrm{~cm}^{2} / \mathrm{C}$, and there was a degree of improvement with sol concentration which again is consistent with the trends in pore size and specific area. These CE values are comparable to leading values reported in the literature (35 - 65 $\mathrm{cm}^{2} / \mathrm{C}$ ) for nanostructured $\mathrm{WO}_{3}$, particularly when using a Li-containing electrolyte $[5,6$, $15,28,40]$. However, what is most remarkable about Fig. 10 is not the slope, but the extent of both the $\mathrm{x}$ and $\mathrm{y}$ axis. In leading electrochromic reports the ultimate charge density values are $<30 \mathrm{mC} / \mathrm{cm}^{2}$ and the optical density does not significantly exceed unity $[6,14,28]$, reflecting the limited degree of intercalation and optical modulation obtained in these systems. However in this work the OD extends well beyond 2 and the charge density exceeds $150 \mathrm{mC} / \mathrm{cm}^{2}$, which is more than double that of the highest value (60 $\mathrm{mC} / \mathrm{cm}^{2}$ ) [40] reported in the literature for a nanostructured tungsten oxide film. Thus, 
the unprecedented $100 \%$ broadband modulation achieved in this work is due to the combination of good intrinsic properties that are maintained over micron length scales to provide the requisite charge density. The exceptional properties displayed in this work are expected to beneficial in related applications of mesoporous tungsten oxide such as photo-catalysis [13], gas sensing [36], and energy storage [41]. For example, as a pseudocapacitor the material produced in this work displays superior performance in terms of its volumetric storage capacity $\left(>350 \mathrm{~F} / \mathrm{cm}^{3}\right)$ and charging ability than a recent leading report [41].

A final important metric for smart window applications is their long term durability [5]. The films produced in this work were subjected to extended cycling tests. Fig. 11a,b displays the optical transmission and the relative changes in coloration efficiency and charge density of a film deposited at $1 \mathrm{C}$ x $6 \mathrm{P}$ through 1000 cycles of modulation between 2 and $3 \mathrm{~V}$ vs. $\mathrm{Li} / \mathrm{Li}^{+}$under constant current density. The films produced at the other sol concentrations displayed comparable performance. The optical transmission in the bleached state remained essentially unchanged at $\sim 100 \%$ throughout the test. In contrast, the transmission level in the colored stated increases rather sharply during the first 50 cycles, and then continues to degrade at a more gradual pace throughout the cycling test. These results can be understood by comparing the changes in coloration efficiency and charge density as shown in Fig. 11b. The CE value degrades by $\sim 15 \%$ during the first 50 cycles, but then stabilizes for the remainder of the test. The reasons for the initial loss are not fully understood at the present time, but it is encouraging that after this initial decline the intrinsic performance remains stable through at least 1000 cycles. In contrast, the charge density declines steadily throughout the test. This behavior was observed 
previously in our nanoparticle-based films [23], and was attributed to the detachment of $\mathrm{WO}_{3}$ nanocrystals into the liquid electrolyte in response to the volume changes associated with intercalation. In principle this would not be a problem in a solid state device, however we also demonstrated that this issue may be mitigated using a few layers of alumina applied through atomic layer deposition without any adverse impact on switching behavior [24]. The stabilization of the coloration efficiency through 1000 cycles demonstrates promising durability for smart window applications. Further work is needed to fully understand the stability behavior in this material.

\section{Conclusions}

In conclusion, we have synthesized mesoporous, nanocrystalline tungsten oxide thin films through ultrasonic spray deposition. The film thickness was directly proportional to the product of the sol concentration and the number of passes under the spray nozzle, facilitating optimization of electrochromic performance. This process adapts standard templated sol-gel chemistry, and by tuning the $\mathrm{WCl}_{6}: \mathrm{P} 123$ ratio and the sol concentration uniform, haze-free films were produced that display an unprecedented $100 \%$ optical modulation across the visible spectrum $(550-950 \mathrm{~nm})$. This exceptional performance is attributed to a combination of good intrinsic quality that is maintained over micron thick length scales, producing specfic charge densities in excess of $150 \mathrm{mC} / \mathrm{cm}^{2}$.

Electrochromic performance, in particular switching speed, scaled with nanostructure and specific surface area, which reached as high a $120 \mathrm{~m}^{2} / \mathrm{g}$. Films displaying $100 \%$ modulation were produced over a wide range of processing and testing conditions, demonstrating the robust nature of the USD approach. These results provide a pathway 
for the production of other mesoporous thin films, as it is expected that many of the solgel chemistries developed previously may be readily transferred to this low cost, scalable manufacturing technique.

\section{Acknowledgements}

This research was founded by National Science Foundation through award DMR0820518 and the Department of Energy under subcontract DE-AC36-08GO28308.

Supporting information available

Photographs of films FTO coated substrates before and after USD synthesis, wide angle XRD scans as a function of sol composition, additional $\mathrm{N}_{2}$ physisorption curves as a function of sol concentration, examples of cross-sectional SEM images used to quantify film thickness, additional CV and transmission measurements as a function of sol concentration, additional transmission spectra as a function of sol concentration.

\section{REFERENCES}

[1] E. S. Lee, S. E. Selkowitz, R. D. Clear, D. L. DiBartolomeo, J. H. Klems, L. L. Fernandes, G. J. Ward, V. Inkarojrit, M. Yazdanian, Advancement of electrochromic windows, California Energy Commission (2006) Report CEC500-2006-052.

[2] G. A. Niklasson, C. G. Granqvist, Electrochromics for smart windows: thin films of tungsten oxide and nickel oxide, and devices based on these, Journal of Materials Chemistry 17 (2007), 127-156.

[3] S. K. Deb, Optical and photoelectric properties and colour centres in thin films of tungsten oxide, Philosophical Magazine 27 (1973), 801-822.

[4] S. K. Deb, Opportunities and challenges in science and technology of $\mathrm{WO}_{3}$ for electrochromic and related applications, Solar Energy Materials and Solar Cells 92 (2008), 245-258.

[5] C. G. Granqvist, Electrochromics for smart windows: Oxide-based thin films and devices, Thin Solid Films, 564 (2014), 1-38. 
[6] S. H. Lee, R. Deshpande, P. A. Parilla, K. M. Jones, B. To, A. H. Mahan, A. C. Dillon, Crystalline $\mathrm{WO}_{3}$ nanoparticles for highly improved electrochromic applications, Advance Materials 18 (2006), 763-766.

[7] R. Deshpande, S. H. Lee, A. H. Mahan, P. A. Parilla, K. M. Jones, A. G. Norman, B. To, J. L. Blackburn, S. Mitra, A. C. Dillon, Optimization of crystalline tungsten oxide nanoparticles for improved electrochromic applications, Solid State Ionics 178 (2007), 895-900.

[8] W. Cheng, E. Baudrin, B. Dunn, J. I. Zink, Synthesis and electrochromic properties of mesoporous tungsten oxide, Journal of Materials Chemistry 11 (2001), 92-97.

[9] L. Meda, G. Tozzola, A. Tacca, G. Marra, S. Caramori, V. Cristino, C. Alberto Bignozzi, Photo-electrochemical properties of nanostructured $\mathrm{WO}_{3}$ prepared with different organic dispersing agents, Solar Energy Materials and Solar Cells 94 (2010), 788-796

[10] L. Yang, D. Ge, J. Zhao, Y. Ding, X. Kong, Y. Li, Improved electrochromic performance of ordered macroporous tungsten oxide films for IR electrochromic device, Solar Energy Materials and Solar Cells 100 (2012), 251-257.

[11] T. Brezesinski, D. Fattakhova Rohlfing, S. Sallard, M. Antonietti, B. M. Smarsly, Highly crystalline $\mathrm{WO}_{3}$ thin films with ordered 3D mesoporosity and improved electrochromic performance, Small 2 (2006), 1203-1211.

[12] J. Yuan, Y. Zhang, J. Le, L. Song, X. Hu, New templated method to synthesize electrochromic mesoporous tungsten oxides, Materials Letters 61 (2007), 11141117.

[13] S. H. Baeck, K. S. Choi, T. F. Jaramillo, G. D. Stucky, E. W. McFarland, Enhancement of photocatalytic and electrochromic properties of electrochemically fabricated mesoporous $\mathrm{WO}_{3}$ thin films, Advanced Materials 15 (2003), 1269-1274.

[14] C.-Y. Kim, M. Lee, S.-H. Huh, E.-K. Kim, $\mathrm{WO}_{3}$ thin film coating from $\mathrm{H}_{2} \mathrm{O}-$ controlled peroxotungstic acid and its electrochromic properties, Journal of SolGel Science and Technology 53 (2010), 176-182.

[15] Y. Zhang, J. Yuan, J. Le, L. Song, X. Hu, Structural and electrochromic properties of tungsten oxide prepared by surfactant-assisted process, Solar Energy Materials and Solar Cells 93 (2009), 1338-1344.

[16] A. Subrahmanyam, A. Karuppasamy, Optical and electrochromic properties of oxygen sputtered tungsten oxide $\left(\mathrm{WO}_{3}\right)$ thin films, Solar Energy Materials and Solar Cells 91 (2007), 266-274.

[17] D. T. Gillaspie, R. C. Tenent, A. C. Dillon, Metal-oxide films for electrochromic applications: present technology and future directions, Journal of Materials Chemistry 20 (2010), 9585-9592.

[18] H. Bisht, H. T. Eun, A. Mehrtens, M. A. Aegerter, Comparison of spray pyrolyzed FTO, ATO and ITO coatings for flat and bent glass substrates, Thin Solid Films 351 (1999), 109-114.

[19] R. C. Tenent, D. T. Gillaspie, A. Miedaner, P. A. Parilla, C. J. Curtis, A. C. Dillon, Fast-switching electrochromic $\mathrm{Li}^{+}$-doped $\mathrm{NiO}$ films by ultrasonic spray deposition, Journal of the Electrochemical Society 157 (2010), H318-H322. 
[20] A. Corma, P. Atienzar, H. Garcia, J.-Y. Chane-Ching, Hierarchically mesostructured doped $\mathrm{CeO}_{2}$ with potential for solar-cell use, Nature Materials 3 (2004), 394-397.

[21] I. E. Rauda, R. Buonsanti, L. C. Saldarriaga-Lopez, K. Benjauthrit, L. T. Schelhas, M. Stefik, V. Augustyn, J. Ko, B. Dunn, U. Wiesner, D. J. Milliron, S. H. Tolbert, General method for the synthesis of hierarchical nanocrystal-based mesoporous materials, ACS Nano 6 (2012), 6386-6399.

[22] A. H. Mahan, P. A. Parilla, K. M. Jones, A. C. Dillon, Hot-wire chemical vapor deposition of crystalline tungsten oxide nanoparticles at high density, Chemical Physics Letters 413 (2005), 88-94.

[23] C.-P. Li, C. A. Wolden, A. C. Dillon, R. C. Tenent, Electrochromic films produced by ultrasonic spray deposition of tungsten oxide nanoparticles, Solar Energy Materials and Solar Cells 99 (2012), 50-55.

[24] C.-P. Li, R. C. Tenent, A. C. Dillon, R. M. Morrish, C. A. Wolden, Improved durability of $\mathrm{WO}_{3}$ nanocomposite films using atomic layer and vapor deposited coatings, ECS Electrochemical Letters 1 (2012), H24-H28.

[25] C.-P. Li, F. Lin, R. M. Richards, C. Engtrakul, A. C. Dillon, R. C. Tenent, C. A. Wolden, Ultrasonic spray deposition of high performance $\mathrm{WO}_{3}$ films using template-assisted sol-gel chemistry, Electochemistry Communications 25 (2012), 62-65.

[26] F. Lin, J. Cheng, C. Engtrakul, A. Dillon, D. Nordlund, R. Moore, T.-C. Weng, K. Williams, R. Richards, In-situ crystallization of high performing $\mathrm{WO}_{3}$-based electrochromic materials and the importance for durability and switching kinetics, Journal of Materials Chemistry 22 (2012), 16817-16823.

[27] C.-P. Li, F. Lin, R. M. Richards, C. Engtrakul, R. C. Tenent, C. A. Wolden, The influence of sol-gel processing on the electrochromic properties of mesoporous $\mathrm{WO}_{3}$ films produced by ultrasonic spray deposition, Solar Energy Materials and Solar Cells 121 (2014), 163-170.

[28] J. Z. Ou, S. Balendhran, M. R. Field, D. G. McCulloch, A. S. Zoolfakar, R. A. Rani, S. Zhuiykov, A. P. O'Mullane, K. Kalantar-zadeh, The anodized crystalline $\mathrm{WO}_{3}$ nanoporous network with enhanced electrochromic properties, Nanoscale 4 (2012), 5980-5988.

[29] J. Szanyi, The origin of haze in CVD tin oxide thin films, Appl. Surf. Sci. 185 (2002), 161-171.

[30] P. C. A. Alberius, K. L. Frindell, R. C. Hayward, E. J. Kramer, G. D. Stucky, B. F. Chmelka, General Predictive Syntheses of Cubic, Hexagonal, and Lamellar Silica and Titania Mesostructured Thin Films, Chemistry of Materials 14 (2002), 32843294.

[31] G. J. Li, S. Kawi, High-surface-area $\mathrm{SnO}_{2}$ : a novel semiconductor-oxide gas sensor, Materials Letters 34 (1998), 99-102.

[32] L. Zhang, J. Y. Ying, Synthesis and characterization of mesoporous niobiumdoped silica molecular sieves, AIChE Journal 43 (1997), 2793-2801.

[33] C. J. Brinker, Y. Lu, A. Sellinger, H. Fan, Evaporation-Induced Self-Assembly: Nanostructures Made Easy, Advanced Materials 11 (1999), 579-585. 
[34] Y.-J. Cheng, J. S. Gutmann, Morphology phase diagram of ultrathin anatase $\mathrm{TiO}_{2}$ films templated by a single PS-b-PEO block copolymer, J. Am. Chem. Soc. 128 (2006), 4658-4674.

[35] W. H. Lai, J. Shieh, L. G. Teoh, I. M. Hung, C. S. Liao, M. H. Hon, Effect of copolymer and additive concentrations on the behaviors of mesoporous tungsten oxide, Journal of Alloys and Compounds 396 (2005), 295-301.

[36] S. Pokhrel, C. E. Simion, V. S. Teodorescu, N. Barsan, U. Weimar, Synthesis, Mechanism, and Gas-Sensing Application of Surfactant Tailored Tungsten Oxide Nanostructures, Advanced Functional Materials 19 (2009), 1767-1774.

[37] F. L. Shang, H. T. Yang, W. L. Lin, L. Gao, Preparation and Electrochromic Properties of Mesoporous $\mathrm{WO}_{3}$ Film, Advanced Materials Research 79-82 (2009), 867-870.

[38] D. R. Rolison, Catalytic nanoarchitectures - The importance of nothing and the unimportance of periodicity, Science 299 (2003), 1698-1701.

[39] P. D. Yang, D. Y. Zhao, D. I. Margolese, B. F. Chmelka, G. D. Stucky, Generalized syntheses of large-pore mesoporous metal oxides with semicrystalline frameworks, Nature 396 (1998), 152-155.

[40] J. Wang, E. Khoo, P. S. Lee, J. Ma, Controlled Synthesis of $\mathrm{WO}_{3}$ Nanorods and Their Electrochromic Properties in $\mathrm{H}_{2} \mathrm{SO}_{4}$ Electrolyte, Journal of Physical Chemistry C 113 (2009), 9655-9658.

[41] C. Jo, J. Hwang, H. Song, A. H. Dao, Y.-T. Kim, S. H. Lee, S. W. Hong, S. Yoon, J. Lee, Block-copolymer-assisted one-pot synthesis of ordered mesoporous $\mathrm{WO}_{3-\mathrm{x}} /$ Carbon nanocomposites as high-rate-performance electrodes for pseudocapacitors, Advanced Functional Materials 23 (2013), 3747-3754. 


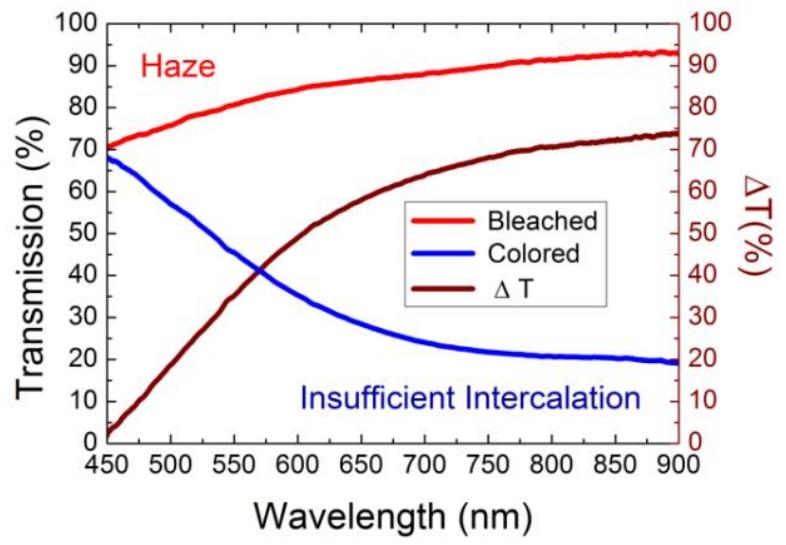

Fig. 1. Optical transmission in the bleached and colored states (left) and the resulting optical modulation (right) as a function of wavelength from $\mathrm{WO}_{3}$ obtained in our previous work [27]. This performance is characteristic of current state-of-the-art $\mathrm{WO}_{3}$, and shows the contributions of haze and insufficient intercalation that limit performance. 


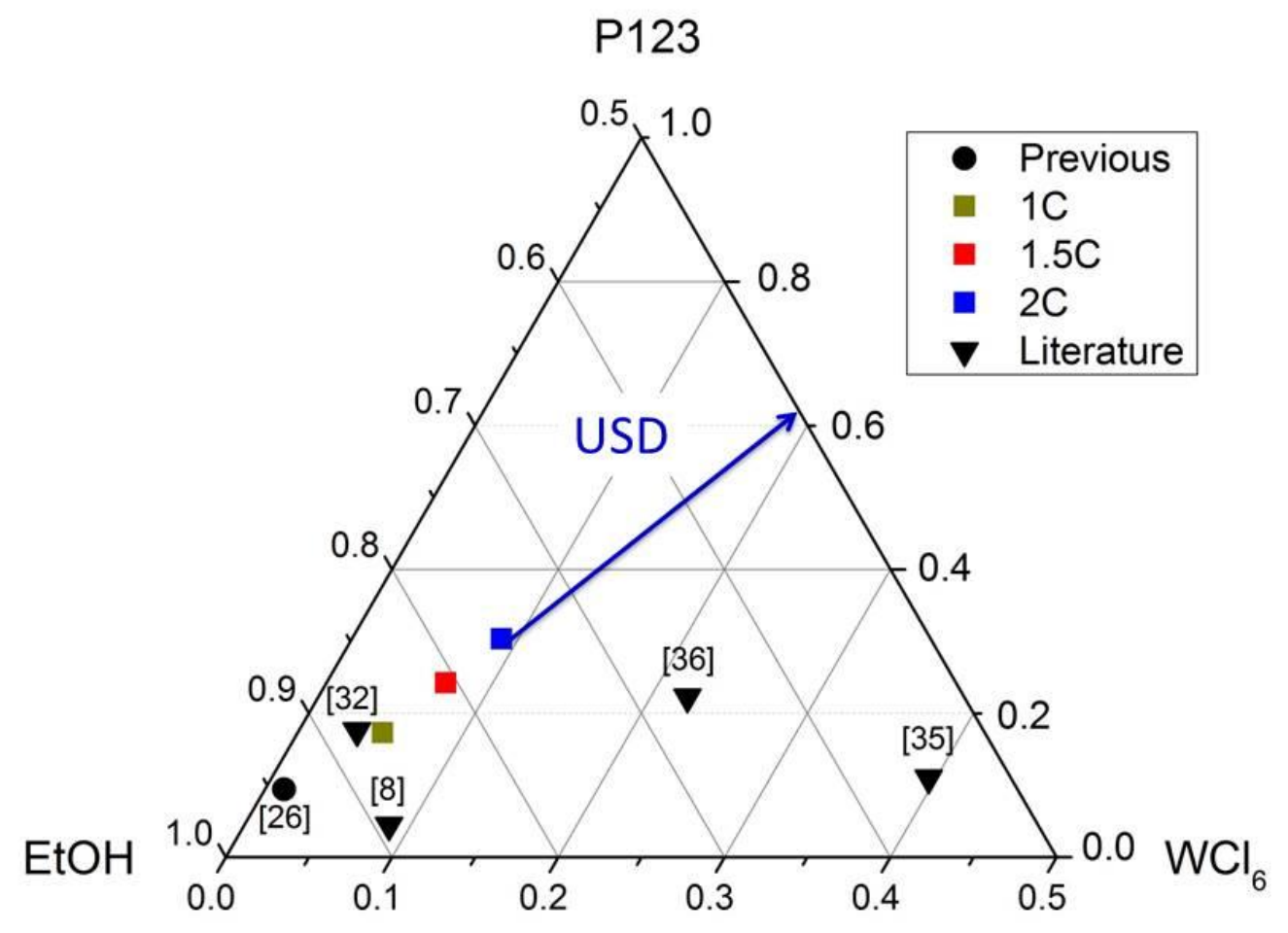

Fig. 2. Ternary phase diagram (wt. \%) comparing the sol compositions explored in this work with those previously reported in the literature. The arrow indicates the concentrating effect that occurs during ultrasonic spray deposition. 


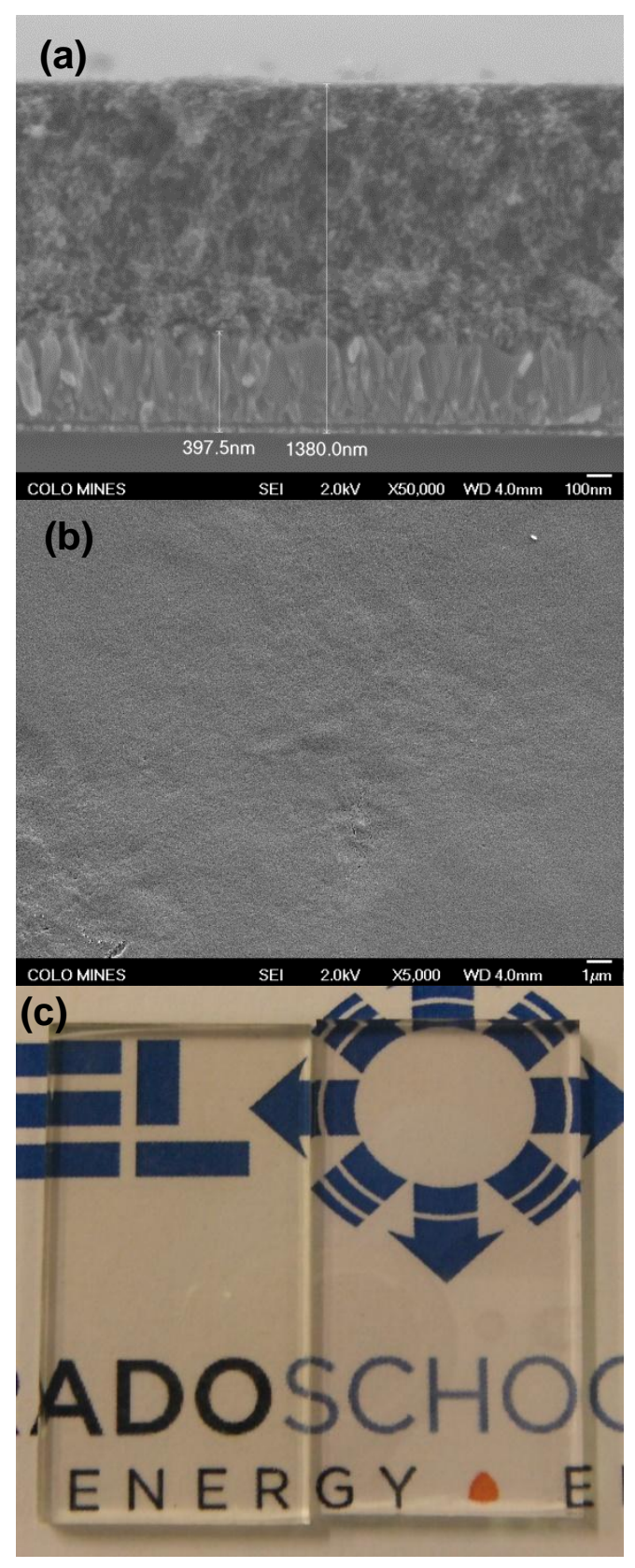

Fig. 3. SEM cross section (a) and plan view (b) images of a mesoporous $\mathrm{WO}_{3}$ film deposited using a 1.5C sol and 4 passes after the calcination step. (c) Photograph of a bare FTO-coated glass substrate (right) and a substrate coated with 1 micron of mesoporous $\mathrm{WO}_{3}$, showing the uniformity of the process and negligible presence of haze in these films. 

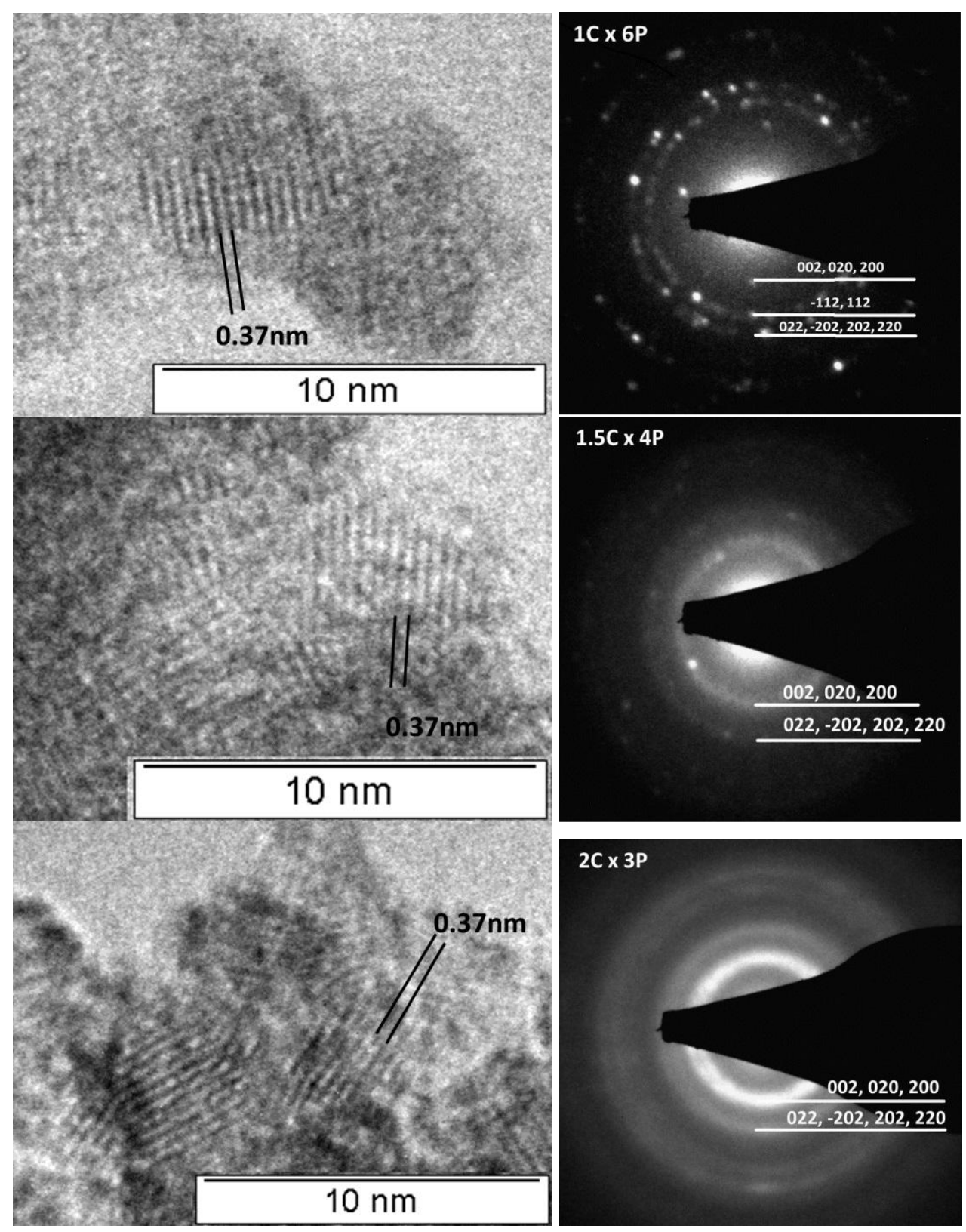

Fig. 4. TEM images (left) and selected area diffraction patterns (right) obtained from $\mathrm{WO}_{3}$ films deposited using sols at $1 \mathrm{C}, 1.5 \mathrm{C}$, and $2 \mathrm{C}$ levels. 

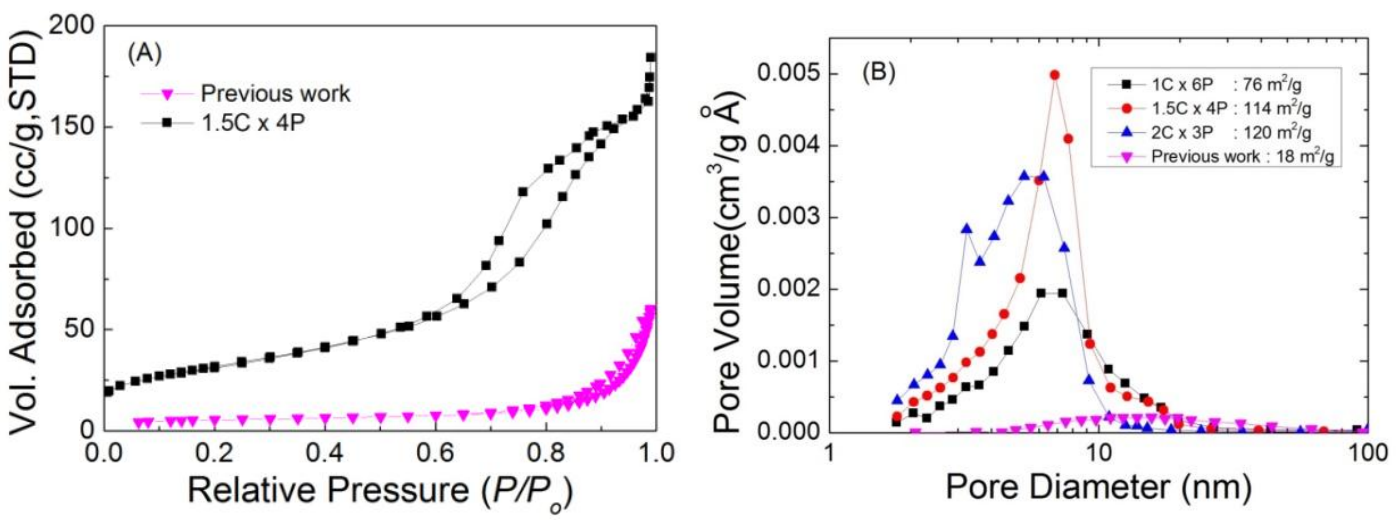

Fig. 5. a) Comparison of $\mathrm{N}_{2}$ physisorption isotherms obtained from mesoporous $\mathrm{WO}_{3}$ produced in this work (1.5 C) and our previous study [27]; b) the resulting pore size distributions and specific surface area obtained from these materials. 
Fig. 6. Plot of the optical modulation (left axis) obtained during cyclic voltammetry as a function of the product of the sol concentration (C) employed and the number of spray passes (P) used to deposit films at sol concentration levels of $1 \mathrm{C}(\Delta), 1.5 \mathrm{C}(\boldsymbol{\square}), 2 \mathrm{C}(\bullet)$. For example, the data at $\mathrm{C} \times \mathrm{P}=6$ was obtained from three films deposited using $1 \mathrm{C} \times 6 \mathrm{P}$, 1.5C x 4P, and 2C x 3P, respectively. The thickness $(\diamond)$ of selected films as measured by SEM cross section is plotted versus the right axis as well as a fit showing the linear relationship between film thickness and the product $\mathrm{C} \times \mathrm{P}$. 


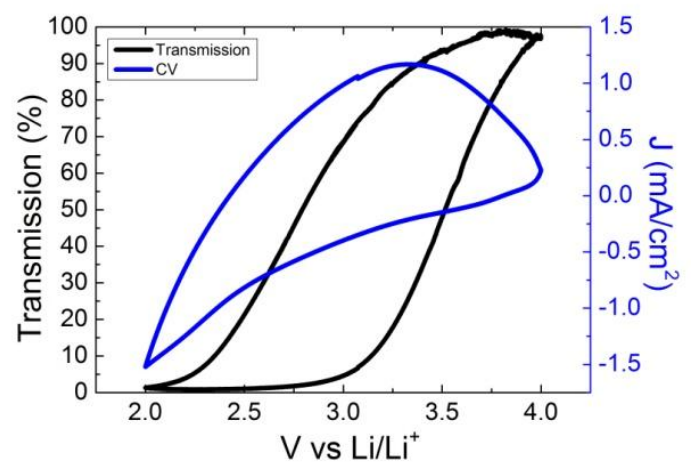

Fig. 7. Cyclic voltammogram (right axis) and the corresponding optical transmission at $670 \mathrm{~nm}$ (left axis) obtained in registry from a film deposited using 1.5C x 4P. 


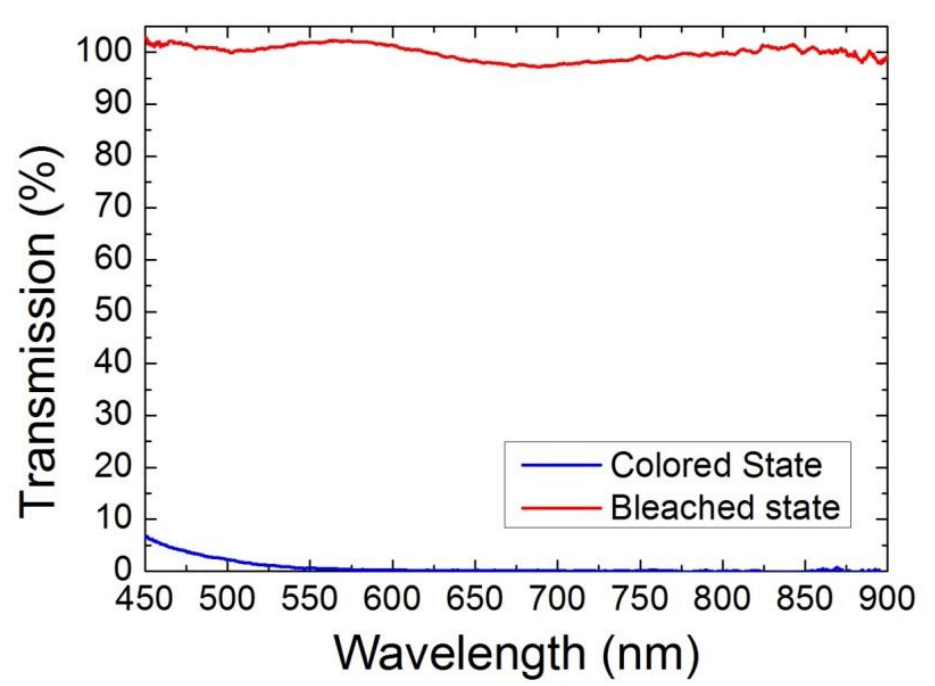

\section{Bleached}

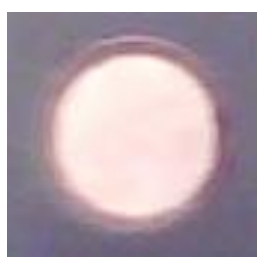

Colored

Fig. 8. Bleached and colored transmission spectra obtained from a film deposited using 1.5C x 4 passes. Insets: Photographs of samples illuminated by a flashlight. 
50

51

52

53

54

55

56

57

58

59

60

61

62

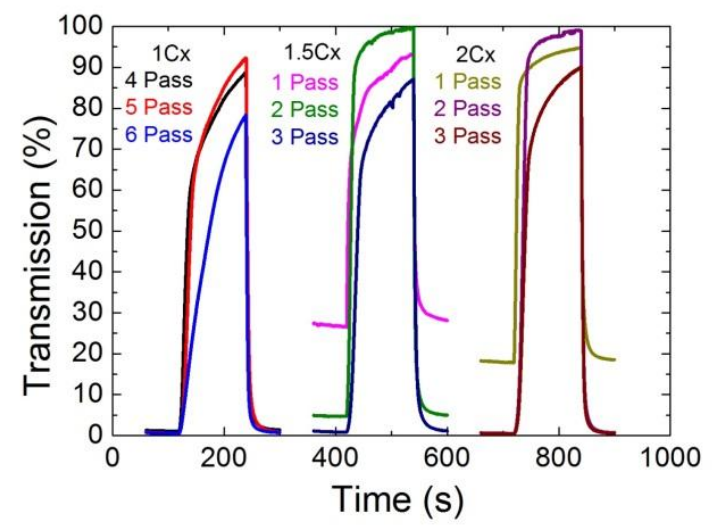

Fig. 9. The dynamic optical response to applied step potentials obtained from films deposited using $1 \mathrm{C} \times 4$ 4, 5, 6 passes, $1.5 \mathrm{C} \times 1,2,3$ passes and $2 \mathrm{C} \times 1,2,3$ passes. 


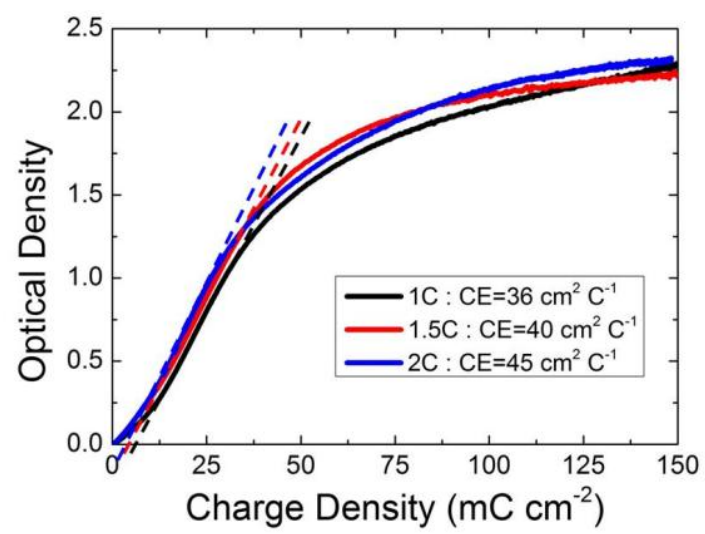

Fig. 10. Plot of optical density vs. charge density for films deposited from sols at the 1C, 1.5C, and $2 \mathrm{C}$ levels. The coloration efficiency $(\mathrm{CE})$ is extracted from the initial slopes and the resulting values are tabulated in the legend. 

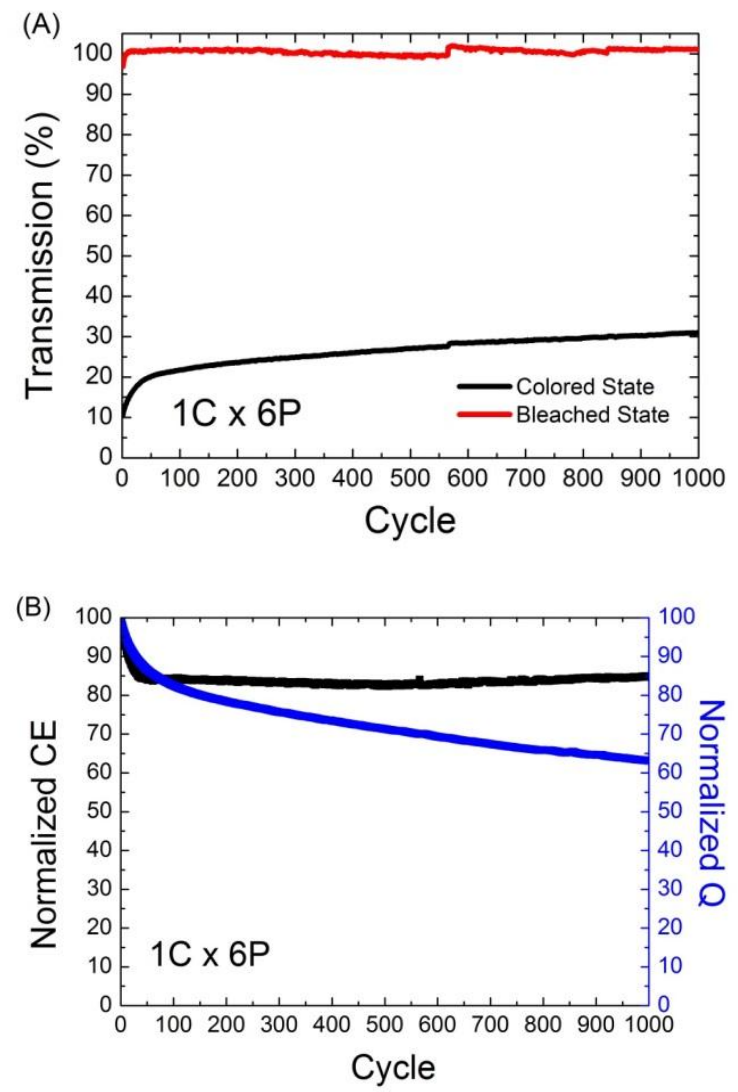

Fig. 11. (a) Optical transmission $(670 \mathrm{~nm})$ in both the bleached and colored states and (b) the changes in coloration efficiency ( $\mathrm{CE}$, left axis) and charge density ( $\mathrm{Q}$, right axis) as function of cycle number. 